\title{
Implementation and Design of Wireless IoT Network using Deep Learning
}

\author{
S.V.Manikanthan ${ }^{2}$, T.Padmapriya ${ }^{2}$ \\ ${ }^{1}$ Director, Melange Academic Research Associates, Puducherry \\ ${ }^{2}$ Managing Director, Melange Academic Research Associates, Puducherry
}

Article HistoryReceived: 10 November 2020; Revised: 12 January 2021; Accepted: 27 January 2021; Published online: 05 April 2021

\begin{abstract}
The Internet of Things (IoT) is a technology and plays a key role in just this process. In designing better cities,embedded devices include this new technology, which the internet is similar to everybody. IoT solutions are spread through different regions, such as agriculture, healthcare, the motor industry, education, and home automation, etc. wireless communication (WC) is now becoming an efficient role for incorporating wireless communicationenvironment, computers, organizations, and governments. WC is a budding technology in IoT, and it involves further learning. This dilemma motivates the authors to suggest a standardized IoT WC system. The formation is subdivided into four tiers, according to the IoT architecture. The initial two steps concentrate on the design of Sensors and gateways; cloud integration is identified in the third stage, the server configuration is discussed in the fourth stage, and cloud integration is listed in the third stage user interface is seen in the fifth stage. Proposed, hence, the solution enables the IoT application developer's work. The design using the clean energy consumption case study. Sensors and Raspberry pi are used in the application to quantify and then provide electricity usage using wireless communication between the devices consumed by the home or building. This provides the client with a rising knowledge of the pattern of power consumption and the usage of electricity using Recurrent Neural Network(RNN).
\end{abstract}

Keywords: Wireless communication(WC), internet of things(IoT), energy consumption, raspberry pi, and recurrent neural network(RNN).

\section{Introduction}

The Internet of Things (IoT) explains the network of touch screen physical objects, apps, and other technologies to enable interacting data over the Internet with other institutions and stakeholders [1][2][3][4]. Real-time analytics, machine learning, asset sensors, and embedded systems, given the likely convergence of scientific advancements, the application has evolved again from the Internet of Things.[1] Embedded systems, wireless sensor networks, control systems, automation (including home and building automation), and others encourage the Internet of Things at the end of the day. Indeed, IoT technology is synonymous with devices in the retail sector. The whole ecosystem, such as smartphones and smart speakers, is discussed in support through one or more common ecosystems, including devices and appliances that support one or more different ecosystems (such as lighting fixtures, thermostats, home management systems, and cameras, and other home appliances) and may even be powered by results.

There are a series of serious concerns about the vulnerabilities of emerging technologies, especially in the areas of safety and privacy, such as industry and confidentiality government movements, including the adoption of policy rules, have also begun to address these concerns.The Internet of Things (IoT) is a mix of natural computer hardware, mechanical and digital devices, objects, animals, or humans with separate UIDs and the needed satellite communications over a network without the need for human-to-human or human-to-computer communications.One of the contents of the Internet a citizen with a heart monitor implant, a farm animal with a biochip transponder, a truck with built-in sensors that require immediate action when the tyre pressure is low, or any other natural or man-made object that can send an Internet Protocol (IP) address and some other natural or man-made object can communicate data over a network.

Web-enabled smart devices, including embedded systems, such as processors, sensors, and communication hardware, are an IoT ecosystem and absorbs, analyses, and acts on data that they collect from their environments. By connecting to an IoT gateway or other edge computer, IoT devices share the sensor data they have collected because data is either sent to the cloud with faster access or application. These devices still link with other similar devices and interact with each other on the specific inputs they seek. The electronics without which to do plenty of job social interaction, while humans can contact the devices forward them reminders or access the data for once to set them up. The protocols used with these web-enabled devices for connectivity, networking, and communication depend heavily on the actual IoT applications deployed. Artificial intelligence (AI) and machine learning are both used in IoT to make data collection safer and more interactive. The IoT helps children to feel and work more cogently, and to have better health. In terms of providing IoT is imperative for smart devices to automate homes, sector. 
IoT simply gives its subscribers a real-time look at how their clients are now doing processes transact business, looking deeper into everything from computer reliability to supply chain operations and logistics. Solutions enable organizations to accelerate operations and decrease operating costs. It also eliminates waste and increases service quality, making the processing and delivery of goods less wasteful, as well as the book stated in electronic transactions. As such, IoT is one of real life's most crucial inventions, and as more people understood the importance of keeping them competitive, embedded devices, it will continue to pick up strength.

The Internet of Things systemic toxicity of online devices and demands the use of billions of data points, all of which need to be supervised. Virtualization defense and IoT privacy are cited as major concerns, as along with its enlarged attack surface.Mirai, a botnet that disrupted domain name server provider Dyn and took down most websites for a longer period, was one of the current IoT attacks in one of the main distributed denial-ofservice (DDoS) attacks ever seen. most prominent recent IoT attacks in 2016. Control to the network was captured by attackers by accessing badly maintained IoT devices. All a hacker has to do is enjoy the benefits of a simple vulnerability to exploit all the set out to find it unusable, because IoT devices are directly tied. Manufacturers who do not consistently — or at all — redesign their apps leave them vulnerable to cybercriminals. The below figure 1 . shows the IoT network.

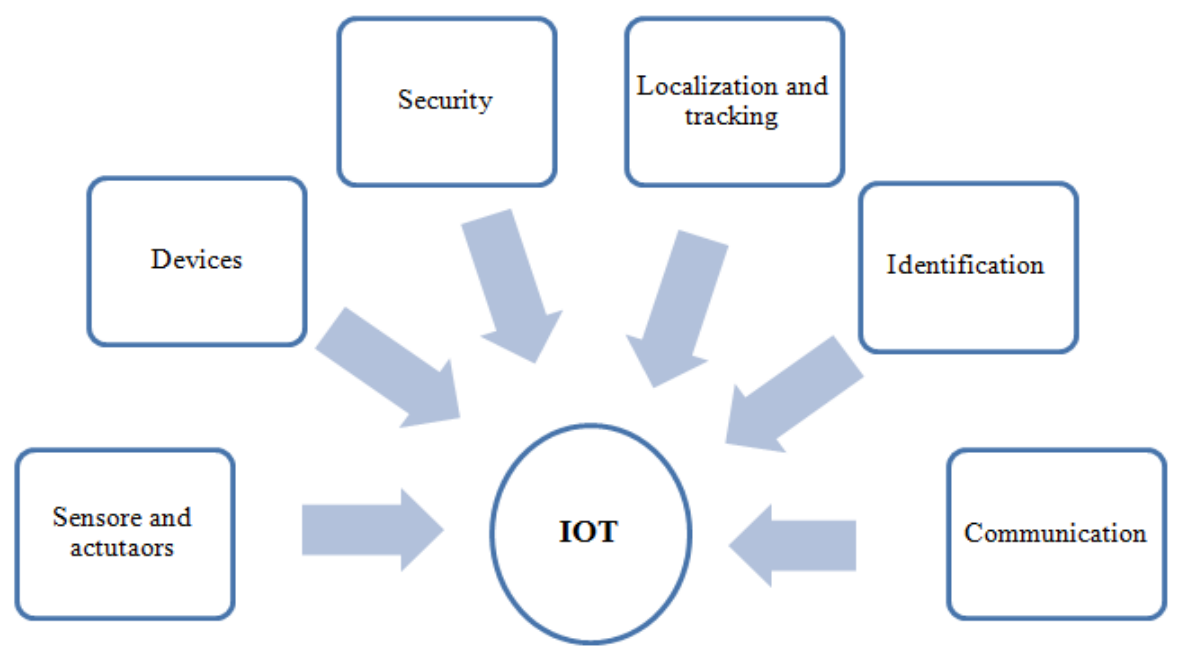

Figure 1. Schematic diagram of IoT network

IoT is a network of things that can be done, also including sensors, smartphones, or any app. Using the Network to make with other devices[5]. Survey method and uploading objects.Over the Internet, so that functions with less energy and fewer resources are automated. Automation, storage, and on-the-fly communication between devices are the critical elements of IoT [6]. The bigger functionality exists under the IoT umbrella. Besides context, healthcare, the construction industry, home automation, livestock, transportation, cars, and trucks, etc. Wireless communication employs microwaves, radio signals, and other wireless media to communicate information between two or more devicesBluetooth, 6LoWPAN, Zigbee, Cellula, BLE, and WiFi[7]. Distinguishing applications are the IoT-used range wireless. The means of cooperation are other technologies. It's Between Tablets. The choice of these, however, helps increase data transmission and framework constraints on growth plates. For illustration, Significant transmission of data and higher transmission range Wi-Fi can be configured over Zigbee.

Also, business and technology constantly ask users to enter their private details that might otherwise be priceless to hackers, including names, dimensions, ages, phone numbers, and even online accounts. Hackers are not the only barrier to network issues; another serious consideration for IoT users is privacy. For example, companies that are manufacturing and selling consumer IoT devices may use the app to manage and sell personal data from users[8]. The next massive development in the new IoT technology is the video game industry, but with the crucial difference that it brings in big improvements incorporate functionality. With a flare in the number of devices connected and the destinations and functions located for the next few years, it's presumed they will succeed. Even then the biggest attribute of the Internet of things is its high effect on many aspects of the idea. The entire years and behaviors of possible buyers. The Internet of Things' most existence based, in both domestic and working industries, as it would be inevitable that private people would think. 
In the first circumstance, Some examples of key application environments in which the Internet of the Internet, the latest paradigm, is Voice control, e-health, assisted living, and better living will a leading role to be assumed shortly educational [9] [10]. Company users could experience the significant judgments in the second case, which are In some of these locations, for example, logistics, the hybrid model of people and materials, automation and automation, productivity of traceable manufacturing and banking industry. The Internet of Things is based on three strongest features:

The "things" (objects).

$>$ The communication networks that connect them.

$>\quad$ The computer systems using data streaming from and to objects

Overall, the Internet of Things is a category of network with some physical objects or objects that connect to a process and decrypt messages with producers, operators, and some other connected devices, embedded with technologies, electronics, sensors, and networking, achieves greater value, aided by clouds, are indeed also inefficient. The limitations of storage, communication capacity, electrical energy are some examples. Similar inefficiencies drive us to mix technology for Mobile Cloud Computing. (MCC) and the Virtual world. Cloud computing upon the internet, as advancing technology, introduces multiple technologies for the optimization of existing infrastructure efficiencies.

Also, mobile Cloud Computing (MCC), another technology, has been upgraded. A current wave of services has flourished over much of the recent years, founded on the "cloud computing" philosophy to provide access to data and data from data information restricting or limiting the need for hardware equipment anywhere at any time. More in general mobile cloud computing is composed of cloud computing applications and network services to make mobile devices resourceful with relation to processing power, memory, storage, capacity, and comprehension of purpose. As a reframing, digital services for banks and corporations can also be known as a mobile cloud. The expansion of e cloud computing is interdisciplinary approaches to cloud computing and mobile computing.

\section{Related Work}

The author Khan, S., \& Yairi, T.[11] proposed that integrated health management and the diagnostic program is a necessary part of the operating lifecycle of the building, amid the developments in modern technological capabilities. This is because it can be used on the framework of up-to-date documentation to classify anomalies, interpret faults, and predicting future states.Data models can be informed through the use of environment descriptions and on-site feedback using machine learning and statistical definitions. When prepared, it is critical to evaluate the logic for data processing on onboard controllers while providing real-time health analysis and assessment.This integration, however, inevitably includes various barriers and risks for the municipality, strengthening the need to overcome this vexing task with current ideas. Characterized by strong advantages in deep learning, immense interest was being gained in the classification of problems with material and feature extraction. It is a growing research field with diverse domains, so its use for system health management applications must be analyzed if it can be used for maintenance, repair, and turnaround program to enhance overall system resilience or other better products. This article presents a comprehensive study of health management in artificial intelligence-based systems with a focus on recent deep learning developments. To demonstrate its ability, fit for purpose and related theories are discussed. Deep learning shows possible benefits for fault identification and prognostics on the background of the assessed work. There are a set of limitations that however, inhibit its public recognition and require further integration advancement. With potential expectations being enumerated, attention is paid to managing these threats.

The author Ching, T., Himmelstein, D. S., Beaulieu-Jones, B. K., Kalinin, A. A., Do, B. T., Way, G. P., ... \& Xie, W.[12] explained that deep learning lays down rules of algorithms for machine learning that can be integrated raw inputs into intermediate feature layers. Recently, across several fields, these algorithms have yielded significant improvements. Biology and medicine are document domains, but the evidence is ambiguous but sometimes confusing. Deep learning techniques can be specifically focused on solving problems in these fields. We investigate deep learning applications for several biomedical challenges, along with the classification of populations, common biological processes, and health professionals, and whether deep learning can transform these tasks or whether particular issues are inherent in the biomedical system. Find out that there's still some need for deep learning reshape following a series of publications, biomedicine or firmly overcome some of the most urgent issues around the world, but promising progress has been made on the previous state of the art. Even though improvements over previous baselines have been quite gradual, findings strongly suggest that deep learning methods can provide a suitable platform to speed up or sustain human learning. Whilst progress in 
linking the prediction of a complex neural network to input features has still been made, and it remains an open challenge to understand how users would view these techniques to understand testable hypotheses about either the system under the systemresearch. Also, in other cases, the small amount of designated training data poses challenges, as do legal and privacy limits on engaging with protected patient information. Alas, we foresee deep learning that encourages bench and bedside improvements with the potential to shape many biology and medical applications.

The author Yoo, S. M, et al..,[13] proposed that the deep learning standards for embedded systems, applications run. Adding ours ongoing project on an improvement and maintenance for deep learning embedded systems, especially for automotive systems. In general, The phase of deep learning networking and communication can be break two steps: training with a large data collection for a data model and data model action with real data. In our system, are concentrate on system development. Try inventing an inference to the engine to address the embedded operational requirements tools. Define the direction of our design and the structure. Preliminary authentic materials were seen.

The author Mardt, A,et al..,[14] described that there is an expanding demand for high-throughput molecular dynamics simulations for computing the 6300, balances, and brief kinetics of biomolecular processes, such as binding of potassium. The compilation into structural properties of numerical coordinates, the reduction of metric instruments, the clustering of a reduced impact on the validity of data are used in current methods the interconversion rates between molecular structures by a Markov state model or different system. This handcrafted approach takes quite a bit of model-based talent, as poor choices can lead to large modeling errors at any particular moment. To create a deep lesson plan over the use of neural networks in molecular kinetics, or VAMP nets, we use the roundabout for Markov processes (VAMP) here.An entire mapping from molecular coordinates to Markov states is interpreted by the VAMP net in a single end-to-end implementation, thus packaging the entire data processing pipeline. Our design \& implementation is close to or better than Markov's state-of-the-art modeling and includes kinetic models of few entitiesand were easily interpretable.

The author Thibaud, M., et al..,[15] explained that the job creation the proliferation of ubiquitous systems is sustained by the progressive acquiescence of the Internet of Things (IoT) devices and their enabling technologies. In high-risk agricultural, health, and safety (EHS) industries, IoT has been shown to have useful outcomes. Human life is at stake here in these industries and to obtain accurate, economical, and usable solutions and their opportunity to manage at a fine granular level and provide rich low-level proof, IoT-based applications are primed. Until 2016, reviewed research focused entirely on the healthcare industry, the food supply chain (FSC), their mining and energy industries (oil \& gas and nuclear), intelligent transport industries, on IoT-based solutions in high-risk EHS industries (e.g. connected vehicles), and their emergency response operations maintenance and infrastructure management are assessed. In high-risk EHS companies, we also highlight IoTrelated issues and challenges. Then conclude by addressing research questions in these industries and predicted dynamics for IoT.

The author An, N. N., Thanh, N. Q., Yanbing, L., \& Wu, F [16] describes that the internet-of- things goods have radically altered life these days, along with the advancements in technology, in particular one of the Smart home (IoT). It is unimaginable not to mention devices in IOT-related futility for emerging technologies, selfdriving cars, and mostly for artificial intelligence. Smart home devices are driven by voice regularly. Therefore, machine learning is also in need of enhancement.Strengthen the classification of concepts to ensure that reliability in the process helps to bring about an evolutionary transition for smart home devices. In the essay, concentrate primarily on processing the human voice independently of the text. In particular, to formulate a Feature Building Machine will include Convolutional Network (CNN) and Software Assistance Vector (SVM). In the classification of speech and frames, which is a prone and computer classification system, SVMs are also used. The study explores the capabilities in speech recognition the combination of Deep Neural Network (DNN) and SVMs provides a structure for engineering smart home systems. The outcomes of the experiment used for the default Voxcelb database, the global sound recognition character over mainstream i-vector or other CNN instruments.

The author Vorakulpipat, C , et al.., [17] explained that in recent years, network security has become a crucial challenge. Massive high penetration of corporate network access devices may expose these applications to serious security consequences. As the use of personal computers keeps shifting to mobile devices and now IoT devices, it is incredibly beneficial to collect more funds and more open outlets for financial and other knowledge. However, the essence and interpretation of IoT, to process facts and business process, vulnerability from time to time, 've changed. An overview of recent IoT security problems, indicators, and concerns is presented in this paper, and three generations of past to future IoT security are checked. However, to process information and 
client requirements, the model and representation of the IoT vulnerability have changed from time to time. A review of the latest report on IoT problems, indicators, and concerns is presented in this paper, and three generations of past to future IoT security are updated.

The author Kataoka, K.,et al..,[18] described that the power of the ability to communicate with IoT is a tough hurdle as the Internet of Things (IoT) improves and progresses. Where whenever end users wish, a large number of IoT devices can be programmed, target others then left unattended and misused. Without improving the performance of an IoT service and its modules, it is difficult to avoid inappropriate connectivity efficiently on edge networks. In this paper, we argue that 1) the identity of IoT applications, computers, and their communications should be validated and recognized by providers of data centers, developers, and network operators, and 2) eliminate a key step from IoT devices in a stable, scalable and fixed manner. This paper proposes a loyalty List that embodies the distribution of trust from wireless stakeholders and promises the autonomous execution of IoT edge traffic management network through all the convergence of blockchains and software-defined networking (SDN). In need to avoid attacks and attacks, the trust list idea automates the doubting, analyzing, and trusting mechanism for IoT services and devices. Justification of method implementation and the trial of the trust list using both public and private pieces chains prove their great skill and recommend rational deployment studies.

\section{Proposed System}

Our unit is slightly parallel, and the passive weights reduce energy consumption and bandwidth. The current works converge efficiently and we receive very enhanced performance. There are also various uses in health, transport, climate, capacity, or structures of the Internet of Things device: sensors, bendable items, such as shoes, glasses, home automation, etc (domotics). It can stream or accumulate IoT data continuously.

As a big data source. Streaming of intelligence points to the outputs. Delivered or seized in tiny intervals of time to be tested promptly and need to study was identified data and/or make fast picks. Data acquisition corresponds to a vast amount of data, databases are used by the hardware, and software systems used are not capable of To purchase, view, process, and process. Such a second step consists and though their criteria for it should be managed differently, the biological reaction is and not the same. After several days of data generation, Big Data's perception analysis, but data analytics streaming insight should be ready in the range may be from a few hundred milliseconds to a fraction of a second.

The fusion and movement of ideas play a critical role in the advancement of IoT document ubiquitous environments. This process is more interesting and indispensable for time-sensitive IoT applications where a timely application is considered necessary to collect all pieces of data for processing for data fusion and thus reinforce feasible method actionable insights. A few other study attempts have considered data analytics streaming it can also be used intended for high computing online services or systems. The analytics for streaming data on these mechanisms are based on data parallelism and rapid under for parallelism. A large dataset is partitioned into these smaller datasets by data parallelism, on which parallel analytics are conditional. They're productive managerially. Refers to Marginal Processing in obtaining a small batch of data for efficient processing in a computation project pipeline. To return a streaming response, they reduce time latency. The knowledge discovery system is not the only alternative possible.

The need for data parallelism and systematic parallelism by having database design streaming near to the data source, i.e. IoT devices or edge devices). Processing is less acceptable than the data size in the source causes it to be easily processed. Then again, adding Speedy IoT system analysis introduces its own challenges shortages in compute, storage, and power capital at the source of data. It is well-known that IoT is also one of the principal sources of high content, although it focuses on assembling a lot of information of insightful to report their frequently captured status, devices to the Internet of their circumstances. Acknowledgment and meaningful retrieval the core utility of patterns this central utility of big data analytics is the enormous raw input data, as it points to for decision-making and understanding formation, higher levels of insights. It is therefore of major importance to derive these data and comprehend Big Data. For few organizations, it is essential because it allows them to reciever core competencies. Hilbert the findings of big data analytics in social sciences are equivalent to those of big data analytics astronomers and astronomy and biology microscope prototypes.

\subsection{Deep Learning}

DL is many other layers of Artificial Neural Networks become based on, supervised or unsupervised learning techniques (ANNs) likely to learn hierarchical embodiments in deep technologies. There are multiple DL 
architectures consists entirely of multiple layers processing. Each layer is capable of creating non-linear responses based on the data from its input layer. This same functionality of DL is imitated by human mechanisms. Brains and signal-processing materials. DL architecture has gained more attention in recent years, comparable to the other generic logistic regression. These methods are perceived by DL Architectures as shallow-structured versions (i.e. a small subset). Although ANNs have had a major increase in 2006, DNNs have had a significant increase in the past decades begin when. presented the existence of networks of deep ideologies. The state-of-the-art potency of this technology has been observed successively. In most other AI industries, including image recognition, image recognition, retrieval, information and search engines, and natural language translation.

The miniature scale another factor contributing to collinearity was training data. Behaviors, also, the computing capability constraint the introduction of productive deeper exploration was prohibited in those days with FNNs. These limits in coding have been overcome picture or video in hardware and the advancement of Graphics Processing Units (GPUs) and general accelerators for hardware. Following the design areas and levels of DL architecture depth effectiveness, as well as hardware progress, DL architecture depth has benefited from advances in DL techniques accurate simulation algorithms, including deep networks.

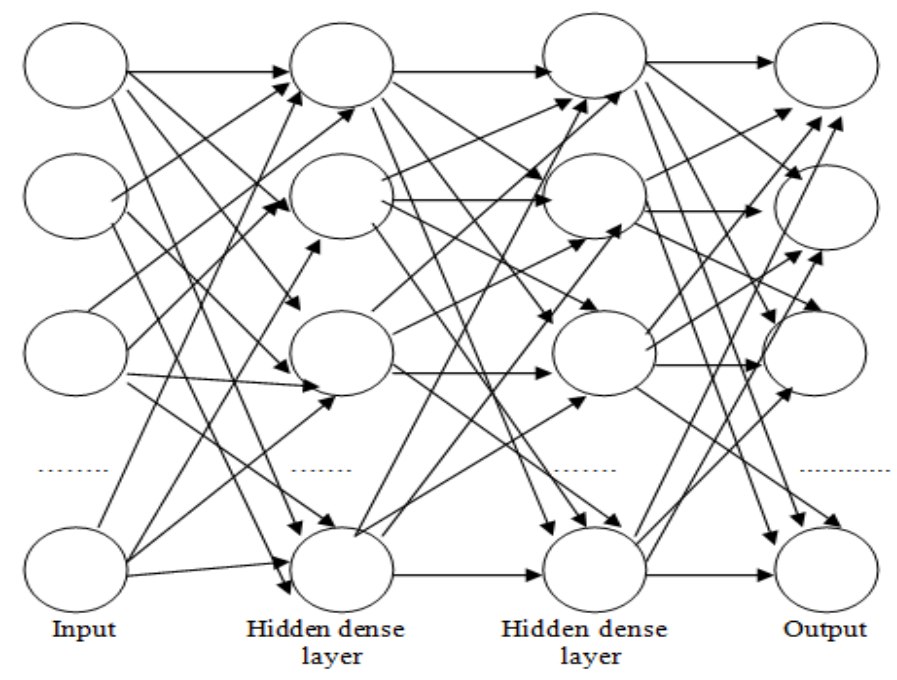

Figure 2. The overall mechanism of deep learning

* Use Rectified Linear Units (ReLUs) as a function to toggle

* Method for dropout presentation

* Random initialization for network weights

* Addressing residual learning networks' impairment of training accuracy

* Solving both of the issues of disappearing gradients and blasting Gradient issue through the implementation and achievement of Long Networks like Short-Term Memory

Compared with conventional ANNs, one strength of DL architectures is that the Modulus of elasticity can learn hidden attributes. From raw materials. Each layer contains a collection of features to train depending on the output. Each layer contains a collection of features to train Focused upon the outputs of the previous layer. The inner-most sphere more complex attributes can be observed, as they multiply and multiply. Recombine features before the layers. This is supported by a comprehensive interface. For example in the case of a face, description model, raw image data for portraits as a vector In its input layer, pixels are fed to a model. More abstract features of each disguised layer can then be comprehended from the outputs of the previous layer, e.g., classify the second layer of the edges describes cream-based lines and the first obscured layer, such as the nose, eyes, the third sheet, etc., utilizes all the previous attributes to create a face. However the identified improvements in the DL models are based on empirical development analyses, and there is no concrete assessment yet analytical basis to address why their shallow peers outperform DL techniques. In particular, there is no definite.

The boundary between networks that are depending on the number of layers raised, deep and shallow. In general two-network neural networks, safeguard layers that recognize the revolutionary new layers or more deep models are called to be training algorithms. Always, recurrent neural networks are believed to have one hidden layer. As deep as the period unrolled into a feasible deep network on the units of the submerged layer is. A DNN consists of a layer of input, two hidden layers, and a layer of output. Each layer contains numerous units named 
neurons receive input information, a weighted neuron performs Summation over its inputs, then the number that reflects passes through an activation function for generating output. Each neuron has a neuron with a weight vector linked with its input size including bias which should be standardized during the period of training. The input layer is defined in the training stage (usually Randomly) weighs and passes on the input training data toward all the next sheet. Weights are also defined by every subsequent layer input to their input and their web client, which acts as the input for the following layer. The final output of the last layer is projected to alter the model's prediction. By computing, the correctness of this estimate is measured by the rate of error between the conflict with bribery and the accurate one.

For the weight of neurons to be dominated by the gradient approximation of the loss function, a Stochastic Gradient Descent optimization algorithm (SGD) is used. The rate of the error is Propagated back down the network to the known input layer as the backpropagation algorithm. Then after balancing the weights on each step, the network repeats this training cycle, until the error rate falls below the inflation rate, the neuron with a threshold. The DNN is trained at this stage and is ready for Inference. DL models fall into three in a broad categorization generative, discriminative, and hybrid definitions Rhythms. Immoral, but not a definite boundaries, commonly, models represent supervised approaches to learning, while unsupervised learning, embraces generative models. Hybrid plans require the characteristics of discriminatory models and models of connectedness.

\subsection{Recurrent Neural Network}

A recurrent neural network (RNN) is a class of artificial neural networks where a controlled graph along a temporal sequence forms ties between nodes. This permits temporal fluid actions to be experienced. RNNs may use their internal state (memory) to process sequences of inputs derived from feedforward neural networks of variable length. This makes them applicable to processes such as unsegmented, attached handwriting recognition, or word recognition. Three individual network segments with a common actual picture are indiscriminately pointed to by the expression "recurrent neural network," where a finite impulse is one and an infinite impulse is the other. Switch code analysis for all network communities. A recurrent finite impulse network is a directed acyclic graph that can be unrolled and combined with a solely feed-forward neural network, though a recurrent infinite impulse network is an acyclic graph that cannot be unrolled.

There are incredibly stored states recurrent networks of both finite experiences and infinite inclinations, and the storage can be probably partially regulated by the neural network. If that introduces holes in time or has feedback loops, the storage may also be replaced by another network or graph. These maintained states, that are otherwise each gated state or gated memory are referred to as part of long short-term memory networks (LSTMs) and gated persistent units. This is also called the Feedback Neural Network (FNN). Basic RNNs are a network separated into successive layers of neuron-like nodes. With a targeted (one-way) connection, each node in a given layer is connected to every other node in the next successive layer. Each node (neuron) has a morning activation of real value. There must be an expandable real-value weight of each contact (synapse). Nodes are either input nodes, or they are (with intelligence gathered outside the network), output nodes (with results), or clandestine nodes (manually configuring the input-to-output data en route).

For supervised learning in discrete-time systems, sequences of real-value input vectors accumulate into the input nodes, one vector at a time. At any given timeframe, and non-input minimum value has its current activation (result) as a nonlinear function of the weighted sum of the activations of all the units which have already been programmedassociated with it. In that implementation of the capability for certain processing facilities, supervisor-given purpose activations can be issued. For goodness' sake, if the input sequence is a speech signal belonging to a spoken digit, the final target output at the end of the sequence might even be a symbol classifying the digit. No author offers aim cues in reinforcement education systems. Instead, a fitness function or reward function is sometimes used to increase the performance of the RNN, which harms its input stream through output glycosidic bonds to public health actuators. This may be used to play a game in which the amount of points gained is closely linked with achievement. As the sum of the deviations from the corresponding activations computed by the network of all target signals, each sequence produces an error. The total error is the sum of the errors of all individual sequences for a training set of alternative sequences.

The problems of gradient depletion and explosion in classical fully related RNNs are tackled by the Independently Recurrent Neural Network (IndRNN). Each neuron in one layer, as historical facts, only receives its past state (instead of wireless connections to all other neurons in this layer) and thus neurons are independent of each other's history. To achieve a long or short-term memory, gradient backpropagation can be managed to prevent gradient depletion and exploding. In the next layers, the cross-neuron content is addressed. With non- 
saturated nonlinear functions such as ReLU, IndRNN can be robustly trained. Deep networks can be trained by using missing connections. The hidden state captures in a serial input the relationship that homeowners might have with each other and it keeps changing at every step, so every input undergoes a different metamorphosis effectively. Compared to a vanilla neural network, the productivity of CNNs and RNNs can be due to the phenomenon of parameter sharing, which is generally a cost-effective way to exploit the interaction between one input item and its surrounding neighbors in a more intrinsic way.

The theory depends on many important regulators in several tasks. Samples such that also need to consider the sequences of inputs, in addition to classifying individual samples. In such a case, architectures do not extend to a feed-forward neural network. Since it does not focus entirely on input and output, the layers do not. To tackle the problem, RNNs have been established in Sequential (e.g., voice or text) or time series problems (data from the sensor) of varying lengths. Detecting the activities of drivers In smart cars, detecting the movement patterns of individuals, some forecasts, and calculations of a household's energy consumption are examples of how it is crucial to schedule RNNs. Production to the input of an RNN.

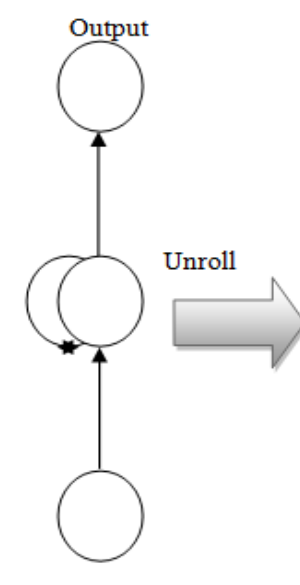

Input

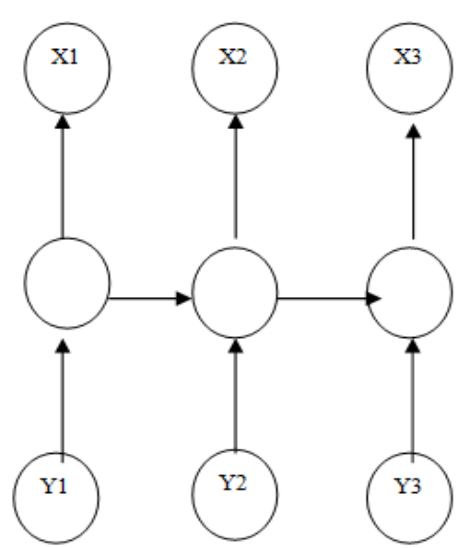

Time

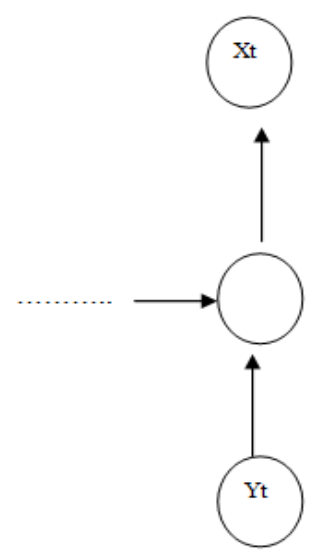

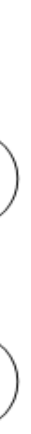

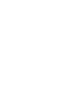

Figure 3. Block diagram of recurrent neural network(RNN)

The existing sample and the previous sample observed are constructed from one sample to that other. In other words, the output of an RNN at stage t-11 in time influences the output of the output at stage t. All deliveries are packaged. A loop of reviews that returns as input the current output. This structure can be described in such a way that each neuron has internal memory in an RNN that controls the results from the previous input computations. For the development of the network, a backpropagation extension Backpropagation whilst also a time algorithm called (BPTT), is being used. Due to the nature of neuron cycles, the original backpropagation should not be used here because it behaves depending on the derivation of error with consideration to the weight. Since we have no stacked-layer model in the upper layer, RNNs. The above diagram 2. shows the block diagram of RNN.

A technique called the origin of the BPTT algorithm is unrolling the RNN so that we can now make the journey forward over geological timescales with a network. Since existing RNNs can be validated as insightful models. They can be interpreted as the prevention of cellular layers between the input layer and the output layer as they are unfolded over time. Given the details, however, the hidden nodes in RNNs are presumed to have had a memory, and rather than a characteristic hierarchical representation. The effectiveness of RNNs. There are many mechanisms for flipping RNNs deeper, including more layers between the hidden layers and the input, packing more hidden layers, and attaching more layers between the output and the input hidden layers.

A recurrent neural network $(\mathrm{RNN})$ is a class of artificial neural networks where relations between nodes are generated by a directed graph along with a temporal series. This helps it to be felt through temporal kinetic theory. RNNs can use their internal state to transmit variable-length sequences of inputs derived from feedforward neural networks (memory). This makes them applicable to policies such as un-segmented, recognition of handwriting, or recognition attached of a word. Two different crowds of the expression "recurrent neural network," where one is a finite impulse and the other is an infinite impulse, indiscriminately corresponds to networks of a similar general structure. Both network groups switch code modeling. 


\section{Results}

Consumers know how to track the use of energy continuously. Where much of the information is lost, a solution such as using less energy can be thinking of. Consuming machines, switching to CFL bulbs, or resource failure inspection. Thus, fuel consumption can be decreased dramatically. The deployed technology is fitted with cameras and is used for information collection. The major focus helping the individual is part of the market monitoring system. To examine the data to understand where energy and cost money has been spent. Reading the data stored in Spark Parquet for Blobs for spectrum sensing and the SQL data warehouse of the cloud, to fulfill the completion of the average final match. This, refining mechanism using the overflow product does not include that on average, takes another 10 to 15 minutes.

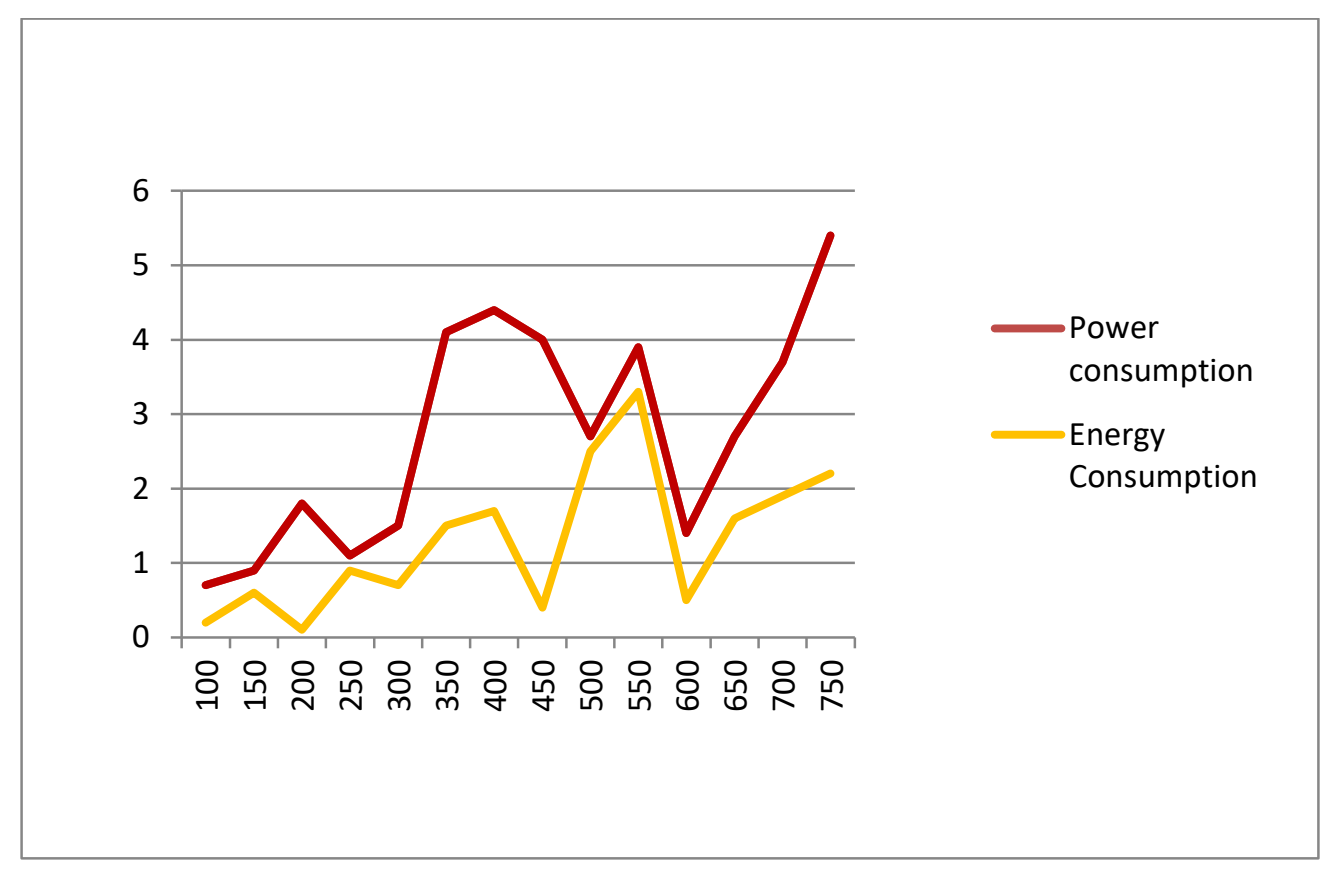

Figure 4. Power and energy consumption using IoT

Compared to an ideal assignment judgment, the device is the function (IDP) that predicts all occupancy for the next hour channels for representatives without any accidents. Then the IDP combines the requests in a data table. the order of their occupancy to the cc outlined in the Occupancy ascending order to enhance the effectiveness of sharing. The average and worst overflow in all test cases the pairs produced by the IDP are $3.73 \mathrm{~s}$ and $36.14 \mathrm{~s}$ compared to. Before using the $10,4.76 \mathrm{~s}$, and $64.48 \mathrm{~s}$ of the developed model predictor for detection. The overloading factor of the holds the ratio of the complete occupancy of the networks used in the movement of representatives is calculated. All the requests are calculated by their collective occupancy. The stronger the increased the versatility of sharing, the greater the overloading factor, as new requests for more overloaded application area outlets are diverted. The overloading consideration of the proposed decision process based on ML is Often superior to IDP, at 1.36 IDP times on great average. Above figure 4 .shows the energy and power consumption of deep learning using an IoT network.

\section{Conclusion}

Deep learning(DL) and the internet of things (IoT) have attracted considerable attention and in recent years, commercial verticals, such trends seem to have such a positive effect on culture, cities, and the atmosphere as these altitudes ranging, and. A chain of proper oversight represents IoT and DL. In which the Internet of things provides information directly that is analyzed. High-level abstraction is formed by DL models. The IoT systems have been fed to the IoT systems for fine-tuning and insight. Resource Creation. The characteristics of the IoT data were validated in this survey. In total, fast IoT and video content data, as well as IoT Big Data, were highlighted as the six essential categories of their regular IoT data generation and analytics. The current opensource frameworks for the development of the DL architecture are required to include main DL architectures used in the Internet of things. 
Reviewing different applications in different fields. The IoT used on the DL was another member of this survey. There were eleven areas of application in which we outlined six foundational services along with five foundational services. The authors' audit provided their DL methodology and use cases with a structure for other researchers to understand by distinguishing fundamental tenet services as well as vertical IoT systems and apply the system reduces to the key components of IoT smart services to their matters. The new DL architecture paradigm on IoT instruments was surveyed and many steps were taken to address this. Introduced were. Fog and cloud infrastructure-based DL to Another element of this survey was to support IoT applications. The difficulties and potential direction of research were also reported in For IoT applications, the trajectory of DL.

\section{References}

1. Rouse, Margaret (2019). "internet of things (IoT)". IoT Agenda. Retrieved 14 August 2019

2. Brown, Eric (20 September 2016). "21 Open Source Projects for IoT". Linux.com. Retrieved 23 October 2016.

3. "Internet of Things Global Standards Initiative". ITU. Retrieved 26 June 2015.

4. Hendricks, Drew. "The Trouble with the Internet of Things". London Datastore. Greater London Authority. Retrieved 10 August 2015.

5. Alkhamisi, A., Nazmudeen, M.S.H., Buhari, S.M.: A cross-layer framework for sensor data aggregation for IoT applications in smart cities. In: 2016 IEEE International Smart Cities Conference (ISC2), pp. 1-6, Sept 2016

6. Chen, Y., Kunz, T.: Performance evaluation of IoT protocols under a constrained wireless access network. In: International Conference on Selected Topics in Mobile Wireless Networking (MoWNeT), pp. 1-7, Apr 2016.

7. Jover, R.P., Murynets, I.: Connection-less communication of IoT devices over LTE mobile networks. In: 12th Annual IEEE International Conference on Sensing, Communication, and Networking (SECON), pp. 247-255, June 2015.

8. About flask. http://flask.pooco.org/docs/0.11/

9. Swan, Melanie (8 November 2012). "Sensor Mania! The Internet of Things, Wearable Computing, ObjectiveMetrics, and the Quantified Self 2.0". Sensor and Actuator Networks 1 (3): 217-253. doi:10.3390/jsan1030217.

10. Mohammad A. Alsmirat; Yaser Jararweh; Islam Obidat; Brij B. Gupta, "Internet of Surveillance: A Cloud supported Large Scale Wireless Surveillance System," the Journal of Supercomputing, Springer, 2016.

11. Khan, S., \& Yairi, T. (2018). A review on the application of deep learning in system health management. Mechanical Systems and Signal Processing, 107, 241-265.

12. Ching, T., Himmelstein, D. S., Beaulieu-Jones, B. K., Kalinin, A. A., Do, B. T., Way, G. P., ...\& Xie, W. (2018). Opportunities and obstacles for deep learning in biology and medicine. Journal of The Royal Society Interface, 15(141), 20170387.

13. Yoo, S. M., Cho, C., Lee, K. H., Park, J., Jin, S., Lee, Y., \& Kim, B. G. (2019, October). Structure of deep learning inference engines for embedded systems. In 2019 International Conference on Information and Communication Technology Convergence (ICTC) (pp. 920922). IEEE.

14. Mardt, A., Pasquali, L., Wu, H., \& Noé, F. (2018). VAMPnets for deep learning of molecular kinetics. Nature communications, 9(1), 1-11.

15. Thibaud, M., Chi, H., Zhou, W., \& Piramuthu, S. (2018). Internet of Things (IoT) in high-risk Environment, Health, and Safety (EHS) industries: A comprehensive review. Decision Support Systems, 108, 79-95.

16. An, N. N., Thanh, N. Q., Yanbing, L., \& Wu, F. (2019, June). Combining Deep Neural Network with SVM to Identify Used in IoT. In 2019 15th International Wireless Communications \& Mobile Computing Conference (IWCMC) (pp. 1145-1149). IEEE.

17. Vorakulpipat, C., Rattanalerdnusorn, E., Thaenkaew, P., \& Hai, H. D. (2018, February). Recent challenges, trends, and concerns related to IoT security: An evolutionary study. In 2018 20th International Conference on Advanced Communication Technology (ICACT) (pp. 405410). IEEE.

18. Kataoka, K., Gangwar, S., \& Podili, P. (2018, February). Trust list: Internet-wide and distributed IoT traffic management using blockchain and SDN. In 2018 IEEE 4th World Forum on the Internet of Things (WF-IoT) (pp. 296-301). IEEE. 